\title{
Changes in transcranial electrical motor-evoked potentials during the early and reversible stage of permanent spinal cord ischemia predict spinal cord injury in a rabbit animal model
}

\author{
MINGGUANG WANG, FANGUO MENG, QIMIN SONG, JIAN ZHANG, CHAO DAI and QINGYAN ZHAO \\ Department of Neurosurgery, Linyi People's Hospital, Linyi, Shandong 276003, P.R. China
}

Received May 19, 2016; Accepted April 7, 2017

DOI: $10.3892 / \mathrm{etm} .2017 .5215$

\begin{abstract}
The present study examined changes in the transcranial electrical motor-evoked potentials (TceMEP) waveform to predict neurological deficits and histopathological changes during the early and reversible stage of different levels of permanent spinal cord ischemic injury in a rabbit animal model. A total of 24 New Zealand rabbits were randomly divided into four groups of 6 rabbits each. Group 1 underwent a ligation of the lumbar artery at three levels (L1-L3), group 2 underwent a ligation of the lumbar artery at four levels (L1-L4) and group 3 underwent a ligation of the lumbar artery at five levels (L1-L5). The sham group contained 6 rabbits and did not receive ligation. TceMEP was recorded within 5 min of ligation and, 2 days later, motor function was assessed and the spinal cords were removed for histological examination. Following spinal cord injury, the relationship between variations in the TceMEP waveform and motor function and pathological damage was analyzed. It was observed that the amplitude of TceMEP began to decrease within 1 min of lumbar artery ligation and that the amplitude stabilized within 5 min. These amplitude changes that occurred within 5 min of different levels of permanent spinal cord ischemic injury were positively related to changes in motor function following recovery from anesthesia and 2 days after ligation. The Pearson correlation coefficient was 0.980 and 0.923 for these two time points, respectively $(\mathrm{P}<0.001)$ In addition, the amplitude changes were positively related to pathological damage, with a Pearson correlation coefficient of $0.945(\mathrm{P}<0.001)$. The results of the present study suggested that amplitude changes in TceMEP are particularly sensitive to ischemia. Ischemia may be detected within $1 \mathrm{~min}$ and the amplitude changes begin to stabilize within 5 min following ligation of the lumbar artery. The use of intraoperative
\end{abstract}

Correspondence to: Professor Qimin Song, Department of Neurosurgery, Linyi People's Hospital, 27 Jiefang Road, Linyi, Shandong 276003, P.R. China

E-mail: 15953911307@163.com

Key words: spinal cord ischemic injuries, evoked potentials, motor, monitoring, intraoperative, rabbits monitoring of TceMEP allows for the detection of spinal cord ischemic injury with no time delay, which may allow for protective measures to be taken to prevent the occurrence of irreversible spinal cord injury.

\section{Introduction}

Surgery on the spine and spinal cord carries a significant risk of injury and postoperative neurological dysfunction. The prevention of paraplegia, a serious complication, resulting from spine and spinal cord surgery has been well documented $(1,2)$. Spinal cord ischemic injury is believed to be the primary reason for neurological dysfunction following spine and spinal cord surgery (3). The role of intraoperative electrophysiological monitoring of the spinal cord functional pathways is to detect spinal cord injury at an early and reversible stage, allowing for the application of protective strategies (4).

Evoked potentials (EPs) are used widely in intraoperative monitoring for the purpose of preventing spinal cord dysfunction (5). Somatosensory EPs (SEPs) were the first to be applied intraoperatively to prevent spinal cord injury (6). However, SEPs reflect only the functional integrity of the spinal sensory pathway, and there have been reports of postoperative paralysis despite the appearance of intact SEPs (7). In addition, SEP recording requires a signal averaging process, which results in a time delay while the neurophysiological physician communicates with the surgeon (8). This lack of real-time feedback prior to intervention may lead to an irreversible spinal cord injury (9). Many of these problems were solved following the adoption of transcranial electrical motor-evoked potentials (TceMEP) intraoperative monitoring. Due to its real-time feedback and proven clinical correlation, there have been many reports of TceMEP leading to protective actions prior to irreversible injury; however, there are still many questions about the use of TceMEP (10).

The present study investigated the relationship between changes in TceMEP amplitude 5 min after spinal cord injury and the severity of the impact on spinal cord function following different degrees of permanent spinal cord ischemic injury in a rabbit animal model. Comparisons were made between TceMEP amplitude changes and pathological changes in neurons of the spinal cord. The aim was to determine a reliable predictor of neurologic deficits at an early and reversible stage of different degrees of permanent spinal cord ischemia. In the 
present experiments, myogenic motor-EPs (MEPs) were generated by TceMEP to help predict the early onset of neurological deficits when intervention is still possible during surgery.

\section{Materials and methods}

Perioperative management. The present study was carried out in strict accordance with the recommendations in the Guide for the Care and Use of Laboratory Animals of the National Institutes of Health. The protocol was approved by the Committee on the Ethics of Animal Experiments of the Union Hospital of Fujian Medical University (Fuzhou, China; permit no. 12-5923).

All animals received humane care. The handling of laboratory animals and their use conformed to the Guidelines for Animal Experiments at Fujian Medical University, Medical Laboratory Animal Management Regulations and other relevant laws and regulations. All experimental animals were fed under the same conditions at the institute, where they had access to food and water ad libitum, and were housed individually in metal cages in a 12-h light/dark cycle at a regulated temperature of $25-26^{\circ} \mathrm{C}$ and relative humidity of $50-65 \%$.

The housing facility maintained national standards, in compliance with the Laboratory Animal-Requirements of Environment and Housing Facilities (GB 14925-2001) (11). If the rabbits appeared to be in extremely poor or moribund condition following surgery, euthanasia was considered and was conducted by intravenous sodium pentobarbital (100 mg/kg; China Langchem, Inc., Shanghai, China).

The management of the laboratory animals conformed to the Laboratory Animal Regulations of the National Science and Technology Commission (12). The animals were monitored according to the experimental design as follows: TceMEP was recorded prior to and within 5 min of ligation; and during the experiment, TceMEP was monitored every $2 \mathrm{sec}$.

The rabbits in the groups with low Tarlov scores (13) were maintained on the premises with sufficient water and food under standard animal house conditions; they could feed themselves with the help of an animal administrator. The paralyzed lower limbs were moved with the help of an animal administrator every $2 \mathrm{~h}$. No incontinence was found in the rabbits in the groups with low Tarlov scores, and all excrement was cleaned in a timely fashion. Those animals with low Tarlov scores of the lower limbs retained some ability of self-care, such as the cleaning of their fur.

All surgery was performed under sodium pentobarbital anesthesia, and all efforts were made to minimize suffering. There was no obvious pain or distress during the experiments, as well as no weight loss, no poor body conditions and no changes in skin or fur conditions. The rabbits were fed under standard animal housing conditions with free access to water and food. Experimental procedures and animal welfare were implemented strictly in accordance with the Use of Laboratory Animals (National Research Council of USA, 1996) and the guide for the care and the related ethical criteria of the Department of Neurosurgery, Fujian Medical University Union Hospital (Fuzhou, China). All measures were made to reduce the number of animals used and to minimize the animals' suffering.
Study design and management. A total of 24 New Zealand white rabbits (14 males and 10 females; weight, $3.0-3.5 \mathrm{~kg}$; age, $11 \pm 1.2$ months), were randomly selected for this experiment. Rabbits were purchased from the Center for Animal Experiments of Hubei Province (Wuhan, China). A sham group (group A) containing 6 rabbits was used to exclude the effects of anesthesia and surgery on EPs and to determine the optimal stimulation intensity. The remaining 18 rabbits in the experimental groups were used to generate models of different levels of permanent spinal cord ischemic injury. The rabbits underwent different levels of serial lumbar artery ligation in a cranio-caudal direction between the renal artery and the aortic bifurcation. In the experimental groups, different levels of lumbar artery ligation were performed at three, four and five levels (groups B, C and D, respectively; $\mathrm{n}=6$ in each group). An observation period of $5 \mathrm{~min}$ was used to detect whether spinal cord ischemia was reflected in TceMEP waveform changes. The sham operation group did not undergo ligation. In the sham operation group, stimulation of different intensities was used to induce TceMEP and to determine the most appropriate stimulation intensity. EPs were recorded before and after the surgery and every $30 \mathrm{~min}$ for $3 \mathrm{~h}$ in the sham group.

Anesthesia management. Intravenous access was established in the marginal ear vein, and anesthesia was infused at a dose of $1 \mathrm{ml} / \mathrm{kg} 3 \%$ pentobarbital sodium and was maintained at $1 / 3-1 / 2$ of the initial dose according to the response of the animals during the experiment. The rabbits were intubated and connected to a respirator to control their breathing during the experiment; nitrous oxide and oxygen at a 2:1 ratio were inhaled. Another intravenous line of lactated Ringer's solution (Henan Huali Pharmaceutical Co., Ltd., Pingdingshan, China) was infused according to the amount of bleeding. During the experiment, body temperature was monitored continuously with a rectal thermometer and was maintained between $38-39^{\circ} \mathrm{C}$ with an electric blanket.

Surgical technique. Rabbits were placed in the supine position after sterile surgical preparations. Additional local anesthesia, containing $0.5 \%$ lidocaine hydrochloride $(1 \mathrm{ml}$; Henan Huali Pharmaceutical Co., Ltd.), was applied to the abdominal wall. A midline abdominal incision was made, and the bowels were removed by turning them to the left and by covering them with wet and heated sterile gauze to reduce fluid and heat loss. After the retroperitoneum was opened and probed, the abdominal aorta and the five lumbar arteries between the renal artery and the aortic bifurcation were exposed. The superior and inferior mesenteric arteries were untouched during the surgery.

Monitoring technique for TceMEPs. Rabbits were placed in a prone position, and the skull was placed in a stereotaxic instrument (Ruanlong Technology Development Co., Ltd., Shanghai, China). The scalp was treated with $1 \%$ lidocaine, and a $3-\mathrm{cm}-$ long incision was cut in the scalp, exposing the skull. The sagittal and coronal sutures of the calvarium were exposed after the periosteum was removed. The stimulating electrodes (Axon Systems, Inc., Hauppage, NY, USA) were fixed on the skull, with the cathode placed in the $\mathrm{C} 4$ position and the anode 
placed in the $\mathrm{C} 2$ position, according to the International $10-20$ system (13). The stimulating electrodes were connected to an EpochXP-2000 electrical stimulator (Axon Systems, Inc.). Silver acupuncture needles were used as recording electrodes and placed subcutaneously into the gastrocnemius muscle of the hind leg. The stimulation parameters were as follows: A stimulation train (three pulses, 120-130 V, 100-msec duration and a 2-msec interstimulus interval) was used to elicit TceMEPs. TceMEPs were also recorded from an upper extremity as a control, and the recording parameters were as follows: Time base, $100 \mathrm{msec}$; bandpass filter of 30-3000 Hz; and amplified 5,000 times. TceMEPs were recorded prior to and within $5 \mathrm{~min}$ following ligation, and the baseline value was determined just prior to the initiation of lumbar artery ligation. The TceMEP amplitude was defined as the voltage range from the most positive to the most negative component. After the baseline values of the TceMEPs were recorded, prepared lumbar arteries were ligated (14) in a cranio-caudal direction.

Evaluation of neurological outcome. The hind limb motor function of all rabbits was assessed after recovery from anesthesia and 2 days after ligation, and the Tarlov score was assessed as follows (15): A score of 1 for spastic paraplegia, cannot move; a score of 2 for paraparesis, slight movements; a score of 3 for paraparesis, powerful movements in hind limbs but not able to stand; a score of 4 for able to stand but unable to walk; and a score of 5 for full recovery, normal walking function. Neurological examination was carried out at the same time by two investigators who were blinded to the groupings and who independently assessed the animals' neurological functions.

Evaluation of pathological outcome. All rabbits were sacrificed with deep intravenous sodium pentobarbital anesthesia $(100 \mathrm{mg} / \mathrm{kg}) 2$ days after surgery. The spinal cord between L2 and L4 was removed and soaked in 10\% paraformaldehyde $/ 0.1 \mathrm{~mol} / 1$ phosphate-buffered saline solution at $4^{\circ} \mathrm{C}$ for $48 \mathrm{~h}$. The spinal cord was sectioned and embedded in paraffin. The experimental slices $(5-\mu \mathrm{m}$ thick) were stained with hematoxylin-eosin and examined by light microscopy for histopathological observation. The neuropathologists were blinded to the experimental groups and observed the destruction of spinal cord motor neurons of the anterior horn. The light microscopy findings were graded on a scale of +3 to 0 , which corresponded to no change, mild, moderate and severe changes, respectively (16).

Statistical analysis. Data were expressed as the mean \pm standard deviation, and TceMEP data were expressed as the median and 10-90th percentiles. Statistical analysis of neurological scores was performed using an unpaired t-test. Multi-group variables were compared using one-way analysis of variance; if this indicated significance, a post hoc test was used to determine which results were significantly different from each other. The relationship between TceMEP and the neurological score was also analyzed using Pearson correlation analysis. Analyses were performed with SPSS v. 17.0 software (SPSS, Inc., Chicago, IL, USA). $\mathrm{P}<0.05$ was considered to indicate a statistically significant difference.
Table I. The amplitude and latency of TceMEP at different times after anesthesia (mean \pm standard deviation).

\begin{tabular}{lcc}
\hline \multirow{2}{*}{$\begin{array}{l}\text { Time after } \\
\text { anesthesia, min }\end{array}$} & \multicolumn{2}{c}{ TceMEP parameter } \\
\cline { 2 - 3 } & Latency, msec $^{\mathrm{a}}$ & Amplitude, $\mu \mathrm{V}^{\mathrm{b}}$ \\
\hline 30 & $13.03 \pm 1.12$ & $5312.67 \pm 1801.85$ \\
60 & $13.12 \pm 1.15$ & $5185.33 \pm 1691.75$ \\
90 & $13.00 \pm 1.15$ & $5202.67 \pm 1680.04$ \\
120 & $13.02 \pm 1.10$ & $5180.00 \pm 1687.67$ \\
150 & $12.98 \pm 1.09$ & $5125.00 \pm 1701.80$ \\
180 & $12.97 \pm 1.12$ & $5086.17 \pm 1490.85$
\end{tabular}

Data are presented as the mean \pm standard deviation. Results were analyzed using analysis of variance, but no significant differences in alatency or ${ }^{\mathrm{b}}$ amplitude were observed between time points. TceMEP, transcranial electrical motor-evoked potential.

Table II. The amplitude and latency of TceMEP at different times before and after surgery.

\begin{tabular}{lcc}
\hline \multirow{2}{*}{ Time } & \multicolumn{2}{c}{ TceMEP parameter } \\
\cline { 2 - 3 } & Latency, $\mathrm{msec}^{\mathrm{a}}$ & Amplitude, $\mu \mathrm{V}^{\mathrm{b}}$ \\
\hline Preoperative & $13.02 \pm 1.10$ & $5300.00 \pm 1816.84$ \\
30 min after surgery & $13.03 \pm 1.07$ & $5213.67 \pm 1679.67$ \\
1 day after surgery & $13.03 \pm 1.11$ & $5013.17 \pm 1587.49$ \\
\hline
\end{tabular}

Results were analyzed using analysis of variance, but no significant differences in alatency $(\mathrm{P}>0.05)$ and ${ }^{\mathrm{b}}$ amplitude $(\mathrm{P}>0.05)$ were observed between different time points before and after surgery. Data are presented as the mean \pm standard deviation. TceMEP, transcranial electrical motor-evoked potential.

\section{Results}

Model success rate. A total of 24 New Zealand white rabbits were used in the present study. During the surgery, 2 rabbits were excluded due to intraoperative hemorrhage or variation. The other rabbits were free to eat after recovering from anesthesia. The success rate of this model was $92.31 \%$.

Control group. In the control group, stimulation with different intensities was used to induce TceMEPs to determine the most appropriate stimulation intensity. When the stimulation intensity was $>120 \mathrm{~V}$, there was no change in the amplitude or latency. The most appropriate stimulation intensity was determined to be $130 \mathrm{~V}$. The EP recordings before and after surgery, and at different time points after the rabbits were anesthetized are demonstrated in Tables I and II.

Experimental groups. Reproducible TceMEPs were found in every rabbit tested. Typical TceMEP wave deflections were described as N1 and P1 (Fig. 1). The baseline amplitude of TceMEP was 5518.00 $\mu \mathrm{V}(3502.60-7903.60 \mu \mathrm{V}, 10-90$ th 


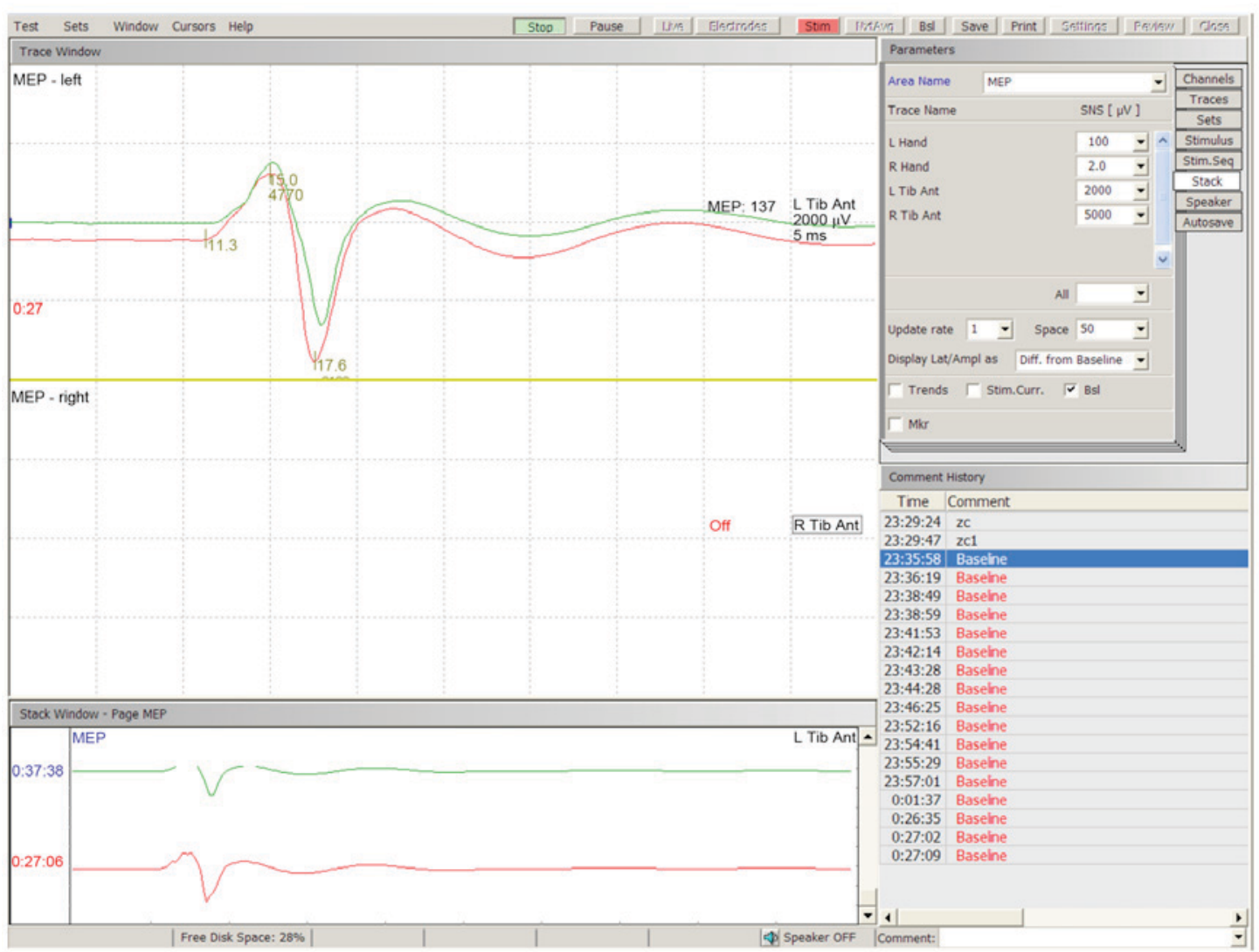

Figure 1. The typical wave of TceMEP. TceMEP latency refers to the duration in msec from stimulation to the first progressive negative deflection. TceMEP amplitude refers to the peak-to-peak amplitude in microvolts (N1-P1). The red graphics reflect the positive and negative baseline waveform before ligation. The green graphics reflect the positive and negative baseline waveform after lumbar arteries were ligated. TceMEP, transcranial electrical motor-evoked potentials.

percentile) in all animals. A decrease in the amplitude of TceMEP was observed following different levels of lumbar artery ligation. In the 3-, 4- and 5-level spinal cord ischemia (SCI) groups, the amplitude decrease was observed within $0.52 \pm 0.08,0.58 \pm 0.15$ and $0.50 \pm 0.12 \mathrm{~min}$, respectively. It took $3.33 \pm 0.38,3.18 \pm 0.41$ and $2.96 \pm 0.46 \mathrm{~min}$, respectively, for the amplitude to stabilize in these groups.

With increasing levels of vascular ligation, the amplitude gradually decreased and eventually disappeared after the ligation of all five lumbar arteries. Once the TceMEP amplitudes stabilized within $5 \mathrm{~min}$ of ligation, the ratios of the amplitudes after the lumbar arteries were ligated compared to the baseline amplitude in groups A, B, C and D were 95.69 $\pm 5.33,63.16 \pm 5.37$, $25.55 \pm 8.44$ and $0.00 \pm 0.00 \%$, respectively (Figs. $2-4$ ).

Functional evaluation. The animals in the sham group demonstrated no neurological deficits after recovering from anesthesia or 2 days after the procedure $(5.0 \pm 0.0$ and $5.0 \pm 0.0$, respectively). In groups $\mathrm{B}, \mathrm{C}$ and $\mathrm{D}$, the neurological scores were $3.80 \pm 0.45,2.00 \pm 0.63$ and $0.00 \pm 0.00$, respectively, after recovering from anesthesia, and $5.0 \pm 0.0,2.67 \pm 0.52$ and $0.40 \pm 0.55$, respectively, 2 days after ligation (data not shown). Rabbits in the sham group demonstrated significantly improved recovery compared with the other SCI groups $(\mathrm{P}<0.05)$ after the animals recovered from anesthesia. There was no significant difference between the sham group and group B 2 days after ligation, whereas there was a significant difference between the sham group and groups $\mathrm{C}$ and $\mathrm{D}(\mathrm{P}<0.05)$.
Histological assessment. In the sham-operated groups, the spinal cord was normal with many intact motor neurons in the anterior spinal horn (Fig. 5). Ischemic damage was observed almost exclusively in the anterior horn spinal cord gray matter in the 3-, 4- and 5-level ligation groups (Figs. 6 and 7). This area contained neuronal necrosis with a typical loss of cytoplasmic structures and eosinophilic cytoplasm, and neuronal apoptotic features, such as apoptotic bodies, chromatin condensation, shrinkage and nuclear fragmentation. The extent of ischemic damage was grossly proportional to the number of ligated lumbar arteries. The pathological score in groups A, B, C and D were $3.00 \pm 0.00,2.40 \pm 0.55,1.33 \pm 0.52$ and $0.20 \pm 0.45$, respectively (data not shown). There were significant differences in pathological score between the sham operation group (group A) and groups $\mathrm{C}$ and $\mathrm{D}(\mathrm{P}<0.05)$, with group $\mathrm{A}$ demonstrating improved pathological scores compared with groups $\mathrm{C}$ and $\mathrm{D}$; however, there was no significant difference between the sham operation group (group A) and group B ( $\mathrm{P}>0.05)$.

The relationship between TceMPE amplitude changes within 5 min following different levels of permanent SCI and motor function. The correlation between the changes in TceMEP amplitude and motor function after recovery from anesthesia was significant $(\mathrm{P}<0.001)$, and the Pearson correlation coefficient was 0.980 . The relationship between the TceMEP amplitude changes and motor function 2 days after lumbar artery ligation was also significant $(\mathrm{P}<0.001)$, and the Pearson correlation coefficient was 0.923 . 


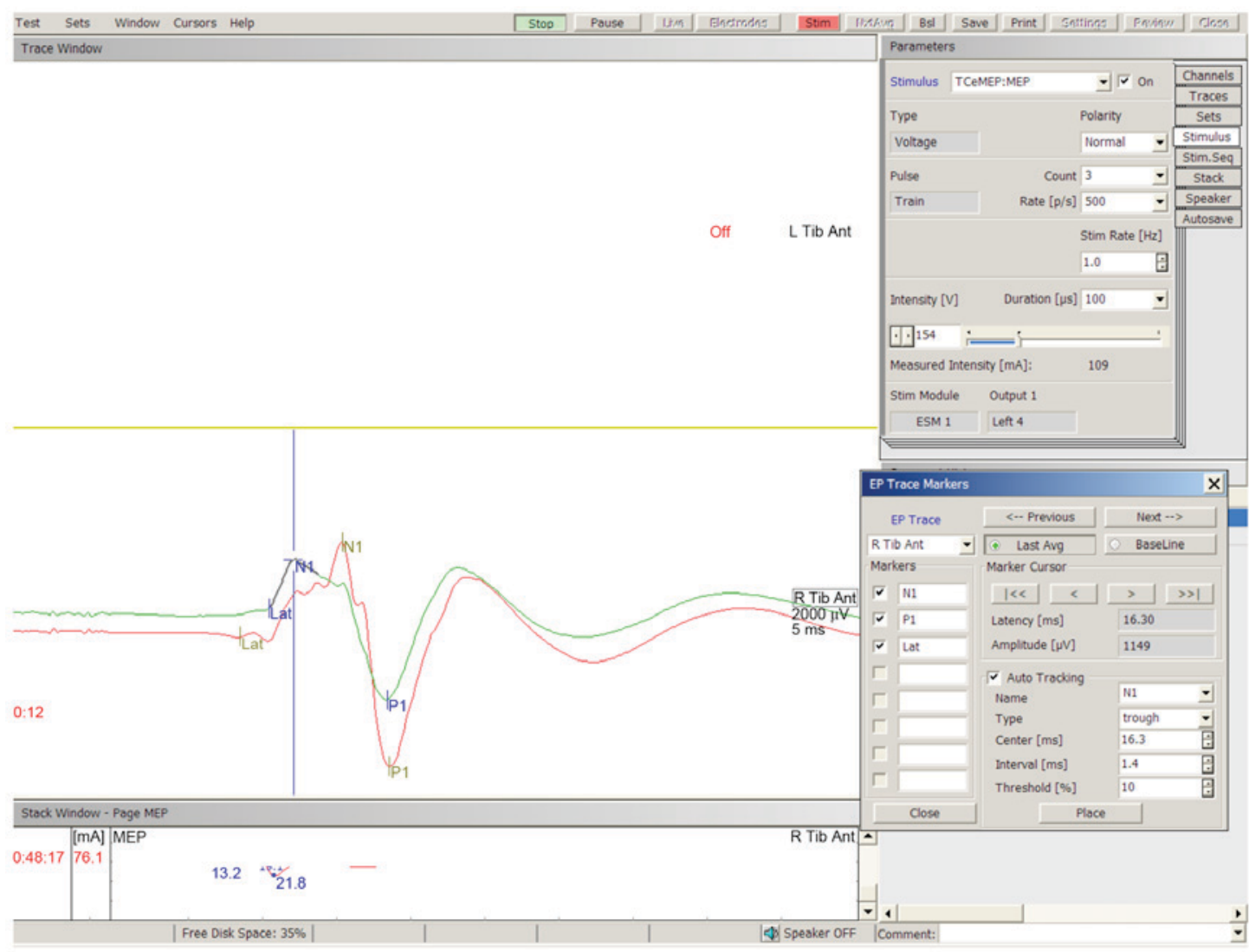

Figure 2. The waveform of transcranial electrical motor-evoked potentials before and after three levels of lumbar arteries were ligated. The red graphics reflect the positive and negative baseline waveform before lumbar artery ligation. The green graphics reflect the positive and negative baseline waveform after the lumbar arteries were ligated.

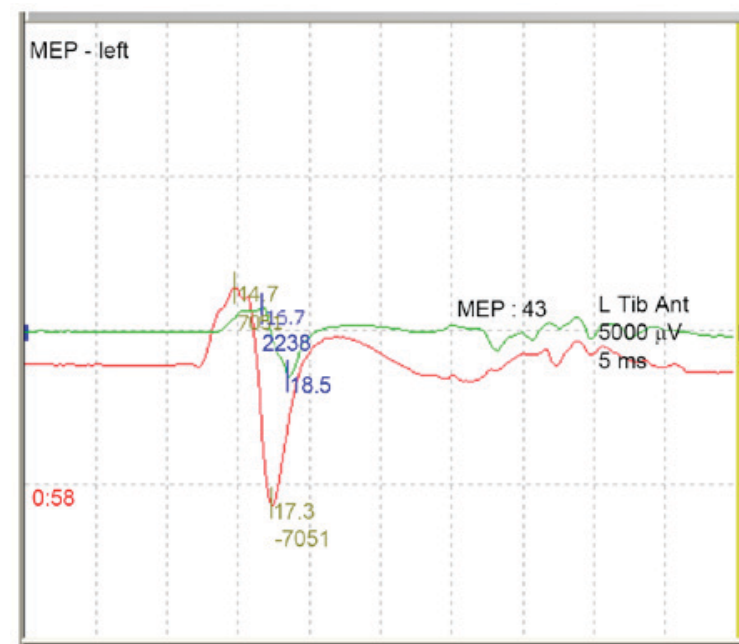

Figure 3. The waveform of transcranial electrical MEP before and after four levels of lumbar arteries were ligated. The red graphics reflect the positive and negative baseline waveform before lumbar artery ligation. The green graphics reflect the positive and negative baseline waveform after the lumbar arteries were ligated. MEP, motor-evoked potential.

The relationship between changes in TceMEP amplitude and the pathological score. The correlation between changes in TceMEP amplitude and the pathological score was significant $(\mathrm{P}<0.001)$, and the Pearson correlation coefficient was 0.945 .

\section{Discussion}

The spinal cord has a particularly complex blood supply network on the surface that includes three main arteries: A single anterior spinal artery and paired posterior arteries. These arteries are joined by a mesh-like pial plexus surrounding the spinal cord. The arterial blood supply of the spine and spinal cord is segmentally provided by lumbar and intercostal arteries branching from the aorta. These rich anastomotic vascular supply network channels offer alternative pathways for supplying the spinal cord with blood and may preserve spinal cord circulation following the ligation of segmental arteries at certain levels (17). The present study demonstrated that, in rabbits, the ligation of lumbar arteries at 1-2 levels does not lead to spinal cord dysfunction, which reveals the presence of a dense and complex collateral arterial network feeding the spinal cord.

Rabbits were used in the present experiment because of their unique lumbar arterial blood supply to the spinal cord from the infrarenal aorta (18). The lumbar artery of rabbits demonstrated fewer variations and is more convenient for ligation, making it particularly suitable as an animal model of spinal cord ischemia, and this model is important for the study of the dynamics of blood supply to the spinal cord and the reaction of the spinal cord to different levels of permanent spinal cord ischemia (19). Furthermore, this model is useful in refining the design and verifying the safety of intraoperative 


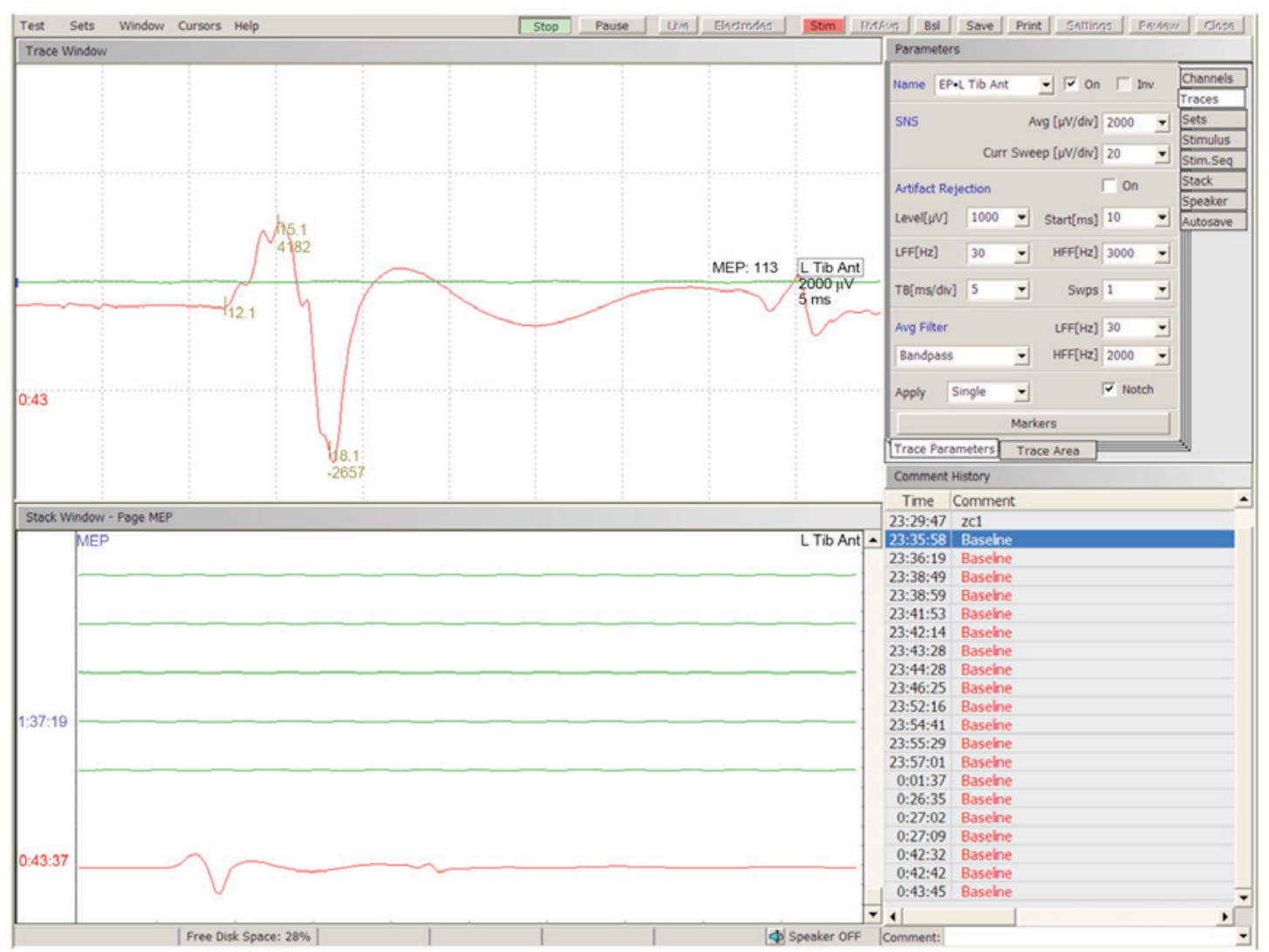

Figure 4. The waveform of transcranial electrical MEP before and after five levels of lumbar arteries were ligated. The red graphics reflect the positive and negative baseline waveform before lumbar artery ligation. The green graphics reflect the positive and negative baseline waveform after lumbar arteries were ligated. MEP, motor-evoked potential.

protective measures in protecting the function of the spinal cord.

With advances in microsurgical and visualization techniques, complicated surgeries on the spine and spinal cord may be performed more easily (20). In spite of these advances, techniques to monitor the functional integrity of the nerve pathways are lacking. This problem has led to many neurosurgeons trying to identify novel neurophysiological monitoring techniques to protect neurological function and decrease the incidence of surgical complications. Cortical (C)SEPs and MEPs have been widely used $(21,22)$. SEPs reflect the ascending sensory pathway of the posterior columns, which are supplied by the paired posterior arteries (23). SEP monitoring has been reported to be a useful and non-invasive method for protecting spinal cord function and detecting spinal cord ischemic injury (24). SEPs have been used frequently because they may be recorded intraoperatively under general anesthesia (25). However, SEPs primarily examine the sensory pathway of the dorsal column, and the specificity and sensitivity of SEPs for monitoring the motor neuron pathways has been questioned $(26,27)$. Furthermore, SEP monitoring cannot be conducted in real-time because each signal is so small that they must be averaged to create an SEP wave (28).

TceMEPs are suitable for the intraoperative monitoring of the spinal cord motor pathway $(29,30)$. TceMEPs reflect the transmission of the spinal cord motor pathway, which is supplied by the anterior spinal artery. Research has indicated that changes in TceMEPs are able to predict motor function (31). TceMEPs are considered a direct reflection of the function of descending motor tracts (32). Recently, research has recommended a unified evaluation of spinal cord function using CSEPs and TceMEPs during surgery (5). The present study examined the value of TceMEP monitoring intraoperatively during spine and spinal cord surgery. A decrease in the amplitude of TceMEP was observed after different levels of lumbar arterial ligation; the 3-,4- and 5-level SCI groups took $0.52 \pm 0.08,0.58 \pm 0.15$ and $0.50 \pm 0.12 \mathrm{~min}$, respectively, to reflect this decrease in amplitude. Therefore, it was concluded that TceMEP is sensitive to spinal cord ischemic damage.

A study by Zivin and DeGirolami (33) has reported classic and traditional animal models for use in evaluating the value of neuroelectrophysiological intraoperative monitoring techniques. There are many serious complications of congestive and ischemic injury in these spinal cord ischemic injury animal models. Ischemia in muscle and peripheral nerves, occurring as a result of clamping of the abdominal aorta, interferes with the interpretation of myogenic TceMEP amplitude and SEP recordings. Some researchers have excluded the effects of muscle and peripheral nerve ischemia in lower extremities as a cause of changes in myogenic TceMEP amplitude by ligating the right femoral artery; in these experiments, myogenic TceMEP amplitudes were preserved for $30 \mathrm{~min}$ (15). However, it remains unclear whether there are any effects that appear after $30 \mathrm{~min}$. The present experiment utilized a novel technique of producing 


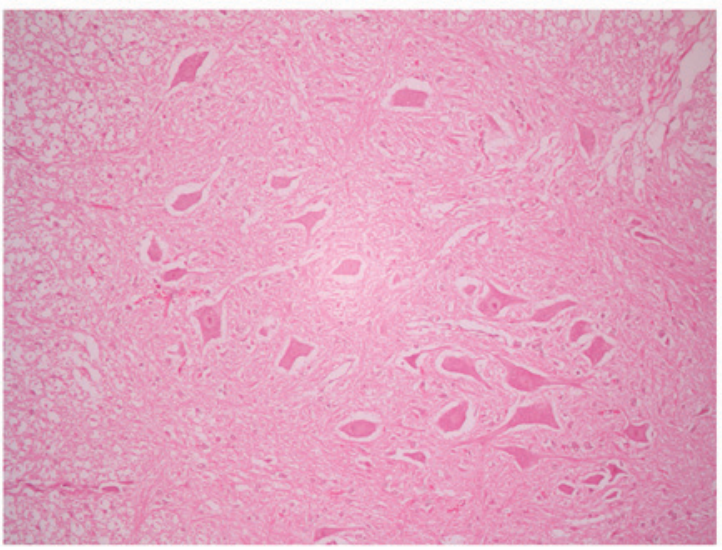

Figure 5. Hematoxylin-eosin stained sections of spinal cord in the control group. Intact motor neurons in the ventral horn were observed. Magnification, x200.

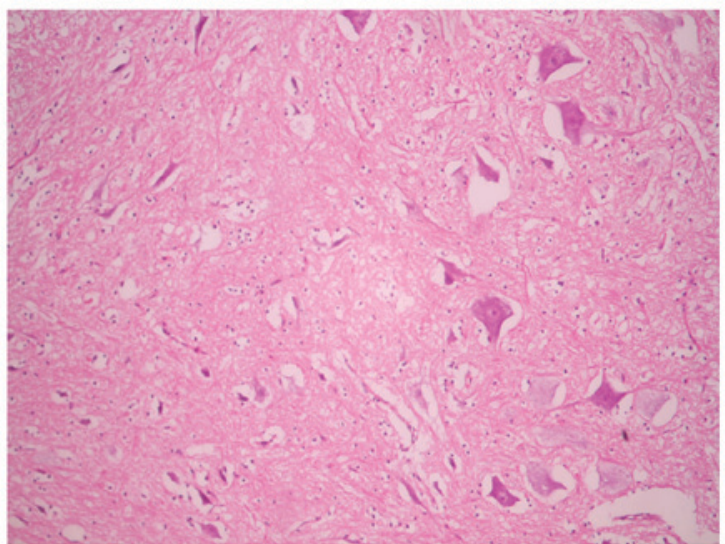

Figure 6. Hematoxylin-eosin stained sections of spinal cord in the 4-level ligation group. Apoptotic and necrotic motor neurons in the most ventral horn were observed. Magnification, x200.

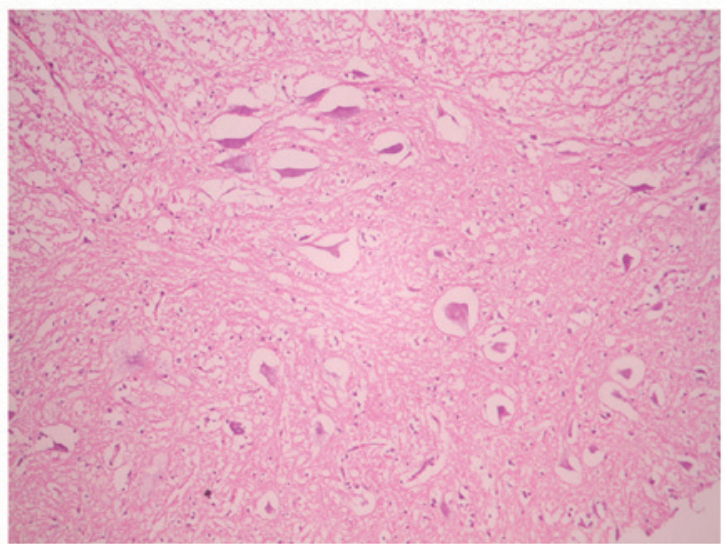

Figure 7. Hematoxylin-eosin stained sections of spinal cord in the 5-level ligation group. Apoptotic and necrotic motor neurons were observed in all ventral horns were observed. Magnification, x200.

permanent spinal cord ischemic injury in rabbits by ligating the lumbar arteries at different levels to evaluate the value of neuroelectrophysiological intraoperative monitoring. The present study did not identify any congestive or ischemic complications that affected the interpretation of myogenic TceMEP amplitude and SEP recordings, particularly in muscle and peripheral nerve ischemia of the lower limbs. As the blood flow to the muscle and peripheral nerves of the lower extremities was unchanged, we believe that this rabbit animal model is ideal for evaluating changes in neurophysiology resulting from different levels of permanent spinal cord ischemic injury.

It is generally believed that 5 min of spinal cord ischemic injury will not result in irreversible damage (34). Therefore, the aim of the present study was to better understand the relationship between changes in MEP at an early and reversible stage of ischemia and the motor function after different levels of permanent spinal cord ischemic injury. The relationship between changes in MEP at an early reversible stage and the pathological damage of anterior horn motor neurons was also investigated. Previous studies have demonstrated that MEPs are highly sensitive to SCI when recorded from muscles in the lower extremities $(35,36)$. A study by de Haan et al $(15)$ demonstrated that myogenic responses disappeared within 2 min after the initiation of arterial occlusion in rabbits. A study by Reuter et al (37) observed that the peripheral nerve responses disappeared within 1 min after the onset of spinal cord ischemia. A study by Kakinohana et al (38) demonstrated that TceMEPs are lost almost instantaneously after the cross-clamping of the thoracic aorta. During the present study, it was observed that the amplitude of MEP began to decrease within $1 \mathrm{~min}$ following the ligation of different levels of lumbar arteries and began to stabilize within $5 \mathrm{~min}$. It was concluded that TceMEP may detect spinal cord ischemia during spine and spinal cord surgery at an early and reversible stage, which would allow surgeons to modify surgical techniques and apply protective measures. A study by Nemoto (39) demonstrated that dogs showed no sign of spinal cord damage when the MEP amplitude was retained at a minimum of $50 \%$ of baseline, and this conclusion is supported by the findings of the present study. When the lumbar arteries are ligated at three continuous levels, motor function was fully restored after 2 days if the amplitude remained at or above $50 \%$ of baseline. However, this function was not restored if the amplitude decreased below $50 \%$.

During resection of intramedullary, subdural and epidural tumors, MEP monitoring has become a true surgical technology (40). Recording and interpretation of MEPs is straightforward and fast. During tumor separation procedures and during tumor resections, spinal cord and nerve roots may be damaged by vascular compromise, compression, traction or electric coagulation hemostasis (40). For detection of such potentially reversible damage, the function of the motor pathways must be assessed continuously with MEP monitoring. The correct prediction of the clinical motor function at a given time during surgery is possible with a very high certainty (41). Loss of muscle MEPs reflects a pattern of MEP change, indicating a reversible injury to the essential motor pathways. Using this information, protective measures may be adopted before irreversible neurological damage is caused, which will allow the surgeon to confidently proceed with a tumor resection. The sensitivity of muscle MEPs for postoperative motor deficits is nearly $100 \%$, with a specificity of $\sim 90 \%$ (41). 
In conclusion, TceMEPs are an ideal spinal cord monitoring method for use during spine and spinal cord surgery, and TceMEP is sensitive to spinal cord ischemic damage. Following different levels of permanent spinal cord ischemic injury, the amplitude changes of TceMEP that occur within 5 min are positively correlated with motor function and pathological damage. The detection of acute spinal cord ischemia by TceMEP monitoring occurs without a time delay, which would allow protective measures to be used in a timely fashion, which may prevent irreversible spinal cord injury.

\section{Acknowledgements}

The present study was supported by funds from the Health Department of Shandong Province of China (grant nos. 2011QW008 and 2015WS0375).

\section{References}

1. Legatt AD, Fried SJ, Amaral TD, Sarwahi V and Moguilevitch M: Loss of lower limb motor evoked potentials and spinal cord injury during the initial exposure in scoliosis surgery. J Clin Neurophysiol 31: e1-e5, 2014.

2. Basoglu H, Kurtoglu T, Cetin NK, Bilgin MD and Kiylioglu N: Assessment of in vivo spinal cord conduction velocity in rats in an experimental model of ischemic spinal cord injury. J Spinal Cord 51: 616-622, 2013

3. Mesquita RC, D'Souza A, Bilfinger TV, Galler RM, Emanuel A, Schenkel SS, Yodh AG and Floyd TF: Optical monitoring and detection of spinal cord ischemia. PLoS One 8: e83370, 2013.

4. Taskiran E, Brandmeier S, Ozek E, Sari R, Bolukbasi F and Elmaci I: Multimodal intraoperative neurophysiologic monitoring in the spinal cord surgery. Turk Neurosurg 27: 436-440, 2017.

5. Jahangiri FR, Sheryar M and Al Okaili R: Neurophysiological monitoring of the spinal sensory and motor pathways during embolization of spinal arteriovenous malformations-propofol: A safe alternative. Neurodiagn J 54: 125-137, 2014.

6. Ando M, Tamaki T, Yoshida M, Kawakami M, Kubota S, Nakagawa Y, Iwasaki H, Tsutsui S and Yamada H: Intraoperative spinal cord monitoring using combined motor and sensory evoked potentials recorded from the spinal cord during surgery for intramedullary spinal cord tumor. Clin Neurol Neurosurg 133: 18-23, 2015.

7. Lesser RP, Raudzens P, Lüders H, Nuwer MR, Goldie WD, Morris HH III, Dinner DS, Klem G, Hahn JF, Shetter AG, et al Postoperative neurological deficits may occur despite unchanged intraoperative somatosensory evoked potentials. Ann Neurol 19: 22-25, 1986.

8. Chang SH, Park YG, Kim DH and Yoon SY: Monitoring of motor and somatosensory evoked potentials during spine surgery: Intraoperative changes and postoperative outcomes. Ann Rehabil Med 40: 470-480, 2016.

9. Pillai JB, Pellet Y, Panagopoulos G, Sadek MA, Abjigitova D, Weiss D and Plestis KA: Somatosensory-evoked potential-guided intercostal artery reimplantation in thoracoabdominal aortic aneurysm surgery. Innovations (Phila) 8: 302-306, 2013.

10. Min HK, Sung K, Yang JH, Kim WS, Jun TG, Lee YT, Park PW and Park BJ: Can intraoperative motor-evoked potentials predict all the spinal cord ischemia during moderate hypothermic beating heart descending thoracic or thoraco-abdominal aortic surgery? J Card Surg 25: 542-547, 2010.

11. Tu XK, Zhang HB, Shi SS, Liang RS, Wang CH, Chen CM, and Yang WZ: 5-LOX inhibitor zileuton reduces inflammatory reaction and ischemic brain damage through the activation of PI3K/Akt signaling pathway. Neurochem Res 41: 2779-2787, 2016.

12. Shi SS, Yang WZ, Chen Y, Chen JP and Tu XK: Propofol reduces inflammatory reaction and ischemic brain damage in cerebral ischemia in rats. Neurochem Res 39: 793-799, 2014.
13. Gürer B, Kertmen H, Kasim E, Yilmaz ER, Kanat BH, Sargon MF, Arikok AT, Ergüder BI and Sekerci Z: Neuroprotective effects of testosterone on ischemia/reperfusion injury of the rabbit spinal cord. Injury 46: 240-248, 2015.

14. LeMaire SA, Ochoa LN, Conklin LD, Widman RA, Clubb FJ Jr, Undar A, Schmittling ZC, Wang XL, Fraser CD Jr and Coselli JS: Transcutaneous near-infrared spectroscopy for detection of regional spinal ischemia during intercostal artery ligation: Preliminary experimental results. J Thorac Cardiovasc Surg 132: $1150-1155,2006$.

15. de Haan P, Kalkman CJ, Ubags LH, Jacobs MJ and Drummond JC: A comparison of the sensitivity of epidural and myogenic transcranial motor-evoked responses in the detection of acute spinal cord ischemia in the rabbit. Anesth Analg 83: 1022-1027, 1996.

16. Ehrlich M, Knolle E, Ciovica R, Böck P, TurkofE, Grabenwöger M, Cartes-Zumelzu F, Kocher A, Pockberger H, Fang WC, et al: Memantine for prevention of spinal cord injury in a rabbit model. J Thorac Cardiovasc Surg 117: 285-291, 1999.

17. Strauch JT, Lauten A, Spielvogel D, Rinke S, Zhang N, Weisz D, Bodian CA and Griepp RB: Mild hypothermia protects the spinal cord from ischemic injury in a chronic porcine model. Eur J Cardiothorac Surg 25: 708-715, 2004.

18. Koçogullari CU, Becit N, Erkut B, Keleş MS, Ceviz M, Ates A, Gündoğdu C, Kaygin MA and Koçak H: Prevention of reperfusion injury of the spinal cord in aortic surgery: An experimental study. Surg Today 38: 237-244, 2008.

19. Mazensky D, Radonak J, Danko J, Petrovova E and Frankovicova M: Anatomical study of blood supply to the spinal cord in the rabbit. Spinal Cord 49: 525-528, 2011.

20. Noh JH, Cho KR, Yeon JY, Seol HJ and Shin HJ: Microsurgical treatment and outcome of pediatric supratentorial cerebral cavernous malformation. J Korean Neurosurg Soc 56: 237-242, 2014.

21. Martin DP, Bhalla T, Thung A, Rice J, Beebe A, Samora W, Klamar J and Tobias JD: A preliminary study of volatile agents or total intravenous anesthesia for neurophysiological monitoring during posterior spinal fusion in adolescents with idiopathic scoliosis. Spine (Phila Pa 1976) 39: e1318-e1324, 2014.

22. James WS, Rughani AI and Dumont TM: A socioeconomic analysis of intraoperative neurophysiological monitoring during spine surgery: National use, regional variation, and patient outcomes. Neurosurg Focus 37: E10, 2014.

23. Stöckl B, Wimmer C, Innerhofer P, Kofler M and Behensky H: Delayed anterior spinal artery syndrome following posterior scoliosis correction. Eur Spine J 14: 906-909, 2005.

24. Ji Y, Meng B, Yuan C, Yang H and Zou J: Monitoring somatosensory evoked potentials in spinal cord ischemia-reperfusion injury. Neural Regen Res 8: 3087-3094, 2013.

25. Mônaco BA, Benício A, Contreras IS, Mingrone LE, Ballester G and Moreira LF: Ischemic preconditioning and spinal cord function monitoring in the descending thoracic aorta approach. Arq Bras Cardiol 88: 291-296, 2007 (In English, Portuguese).

26. Gunnarsson T, Krassioukov AV, Sarjeant R and Fehlings MG: Real-time continuous intraoperative electromyographic and somatosensory evoked potential recordings in spinal surgery: Correlation of clinical and electrophysiologic findings in a prospective, consecutive series of 213 cases. Spine (Phila Pa 1976) 29: 677-684, 2004.

27. Wiedemayer H, Sandalcioglu IE, Armbruster W, Regel J, Schaefer $\mathrm{H}$ and Stolke D: False negative findings in intraoperative SEP monitoring: Analysis of 658 consecutive neurosurgical cases and review of published reports. J Neurol Neurosurg Psychiatry 75: 280-286, 2004.

28. Stecker MM: A review of intraoperative monitoring for spinal surgery. Surg Neurol Int 3 (Suppl 3): S174-S187, 2012.

29. Clark AJ, Ziewacz JE, Safaee M, Lau D, Lyon R, Chou D, Weinstein PR, Ames CP, Clark JP III and Mummaneni PV: Intraoperative neuromonitoring with MEPs and prediction of postoperative neurological deficits in patients undergoing surgery for cervical and cervicothoracic myelopathy. Neurosurg Focus 35: E7, 2013.

30. Szelényi A, Heukamp C, Seifert V and Marquardt G: S100B, intraoperative neuromonitoring findings and their relation to clinical outcome in surgically treated intradural spinal lesions. Acta Neurochir (Wien) 156: 733-739, 2014.

31. Simó M, Szirmai I and Arányi Z: Superior sensitivity of motor over somatosensory evoked potentials in the diagnosis of cervical spondylotic myelopathy. Eur J Neurol 11: 621-626, 2004. 
32. Kobayashi S, Matsuyama Y, Shinomiya K, Kawabata S, Ando M, Kanchiku T, Saito T, Takahashi M, Ito Z, Muramoto A, et al: A new alarm point of transcranial electrical stimulation motor evoked potentials for intraoperative spinal cord monitoring: A prospective multicenter study from the spinal cord monitoring working group of the Japanese Society for spine surgery and related research. J Neurosurg Spine 20: 102-107, 2014.

33. Zivin JA and DeGirolami U: Spinal cord infarction: A highly reproducible stroke model. Stroke 11: 200-202, 1980.

34. de Haan P, Meylaerts SA, Lips J and Jacobs MJ: Development of spinal cord ischemia after clamping of noncritical segmental arteries in the pig. Ann Thorac Surg 68: 1278-1284, 1999.

35. Legatt AD: Current practice of motor evoked potential monitoring: Results of a survey. J Clin Neurophysiol 19: 454-460, 2002 .

36. Liu LY, Callahan B, Peterss S, Dumfarth J, Tranquilli M, Ziganshin BA and Elefteriades JA: Neuromonitoring using motor and somatosensory evoked potentials in aortic surgery. J Card Surg 31: 383-389, 2016.

37. Reuter DG, Tacker WA Jr, Badylak SF, Voorhees WD III and Konrad PE: Correlation of motor evoked potential response to ischemic spinal cord damage. J Thorac Cardiovasc Surg 104: 262-272, 1992
38. Kakinohana M, Abe M, Miyata Y, Oshiro M, Saikawa S, Arakaki K, Kuniyoshi Y and Sugahara K: Delayed response of transcranial myogenic motor-evoked potential monitoring to spinal cord ischemia during repair surgery for descending thoracic aortic aneurysm. J Anesth 22: 304-307, 2008.

39. Nemoto M: Animal experiment on spinal cord ischemia with evoked potentials by transcranial electrical stimulation. J Juntendo Igaku 43: 586-598, 1998

40. Kim DG, Son YR, Park YS, Hyun SJ, Kim KJ, Jahng TA, Kim HJ and Park KS: Differences in multimodality intraoperative neurophysiological monitoring changes between spinal intramedullary ependymoma and hemangioblastoma. J Clin Neurophysiol 33: 120-126, 2016

41. Kothbauer KF: Intraoperative neurophysiologic monitoring for intramedullary spinal-cord tumor surgery. Neurophysiolo Clin 37: 407-414, 2007. 